\title{
Sympatric coexistence of sibling species Harmonia yedoensis and $H$. axyridis (Coleoptera: Coccinellidae) and the roles of maternal investment through egg and sibling cannibalism
}

\author{
NAOYA OSAWA ${ }^{1}$ and KAZUNORI OHASHI ${ }^{2 *}$ \\ ${ }^{1}$ Laboratory of Forest Ecology, Graduate School of Agriculture, Kyoto University, Kyoto 606-8502, Japan; \\ e-mail: osawa@kais.kyoto-u.ac.jp \\ ${ }^{2}$ Laboratory of Ecological Information, Graduate School of Agriculture, Kyoto University, Kyoto 606-8502, Japan
}

Key words. Coccinellidae, coexistence, Harmonia axyridis, Harmonia yedoensis, sibling cannibalism, sibling species

\begin{abstract}
The sibling species $H$. yedoensis Takizawa coexists sympatrically and simultaneously with $H$. axyridis only on pine trees in Japan. To elucidate the mechanisms enabling coexistence of these two sympatric sibling species, a laboratory experiment was performed that focused on differences in their maternal investment through eggs and the role of sibling cannibalism. The egg size (volume) of $H$. yedoensis was $24.91 \%$ larger than that of $H$. axyridis. Cluster size in $H$. axyridis was significantly larger than that in $H$. yedoensis; however, the total number of eggs and oviposition cost (by volume) per female in $H$. yedoensis were not significantly different from those in H. axyridis, although total number of clusters tended to be slightly higher in H. yedoensis than in $H$. axyridis. The percentage of undeveloped eggs per cluster in $H$. yedoensis was not significantly different from that in $H$. axyridis, whereas the percentage of developed eggs with delayed hatching per cluster was significantly larger in $H$. yedoensis than in $H$. axyridis. Moreover, the cost of sibling cannibalism per hatched larval cluster in H. yedoensis (worth 4.43 sibling eggs) was 3.36 times larger than that in $H$. axyridis. Therefore, maternal investment through egg and sibling cannibalism in developed eggs with delayed hatching are more intense in H. yedoensis than in H. axyridis, implying a higher larval survival rate through higher ability of prey capturing at the first instar. The results in this study suggest that the higher survival rate and accelerated development in $H$. yedoensis by the two maternal investments, i.e., a large egg and intense sibling cannibalism of developed eggs with delayed hatching, may play an important role in sympatric coexistence with the aggressive aphidophagous ladybird beetle $H$. axyridis.
\end{abstract}

\section{INTRODUCTION}

Species can be defined as sets of organisms that can mate with each other and produce viable grandchildren, as sets of organisms resembling each other morphologically, or as DNA sequences that act as evolutionarily independent units (e.g., Sterns \& Hoekstra, 2000). Generally, it is known that three closely related species concepts exist: biological, ecological, and phenetic species (e.g., Ridley, 2004). Some biologists suggest that the phenetic species concept, which defines species in general by shaped phenetic attributes, has serious theoretical defects rendering it ambiguous and typological theories of species are also rejected (e.g., Ridley, 2004). The phenetic species concept is based on the premise that phenetic differences among species largely contribute to their reproductive isolation. However, sibling species, as pairs of species that differ reproductively but not morphologically, illustrate that phenetic and reproductive units do not necessarily coincide (Ridley, 2004).

The two ladybird beetles Harmonia axyridis Pallas and H. yedoensis Takizawa (Coleoptera: Coccinellidae) are good examples of sibling species. $H$. axyridis is distributed in the northeast part of Asia and its biology has been studied extensively (e.g., ecological genetics, ecology, systematics, and applied entomology; e.g., Hodek \& Honěk, 1996). H. axyridis has recently been imported to North America, Canada, and Europe, mainly for biological control (e.g., Snyder \& Evans, 2006), where it has had a large impact on the native aphidophagous guilds: Some native ladybird species have disappeared and biodiversity of some aphidophagous community has decreased following invasion by $H$. axyridis, which is an aggressive species. Intense intra-guild predation by $H$. axyridis has been inferred by several authors (e.g., Colunga-García \& Gage, 1998; Yasuda \& Ohnuma, 1999; Adriaens et al., 2003; Brown, 2003; Koch, 2003; Yasuda et al., 2004; Snyder \& Evans, 2006).

Adults of $H$. axyridis are very mobile with high prey searching ability, resulting in concentrated adult arrivals and oviposition in suitable habitats (Osawa, 2000). Larval mortality is a key-factor in $H$. axyridis population dynamics (Osawa, 1993) and the survival rates of early instars are low (Osawa, 1992) mainly because of their low ability to capture aphids (Kawai, 1978). Sibling cannibalism occurs in more than $90 \%$ of egg batches and increases the survival rate of cannibals through unequal resource allocation (Osawa, 1989). Both undeveloped eggs and developed eggs with delayed hatching play an important role in maternal investment, resulting in a high

\footnotetext{
* Present address: Department of Vector Ecology and Environment, Institute of Tropical Medicine, Nagasaki University, Nagasaki 852-8523, Japan.
} 
survival rate and accelerated development of first instars (Osawa, 2002, 2003). Therefore, sibling cannibalism at hatching plays an important role in the life history of $H$. axyridis in natural populations (Osawa, 1993). Moreover, strong density-dependence has been observed in $H$. axyridis populations, caused partly by cannibalism. Egg and pupal cannibalism by larvae have been observed but not egg parasitism, and adults, and maybe eggs as well as larvae, are not eaten heavily by other predators (Osawa, 1993). These stabilizing and persistent self-regulatory mechanisms in $H$. axyridis populations, based on densitydependent mortality and sibling cannibalism at hatching, promote survival in temporally and patchily distributed habitats (Osawa, 1993, 2000), and may be important in enabling $H$. axyridis to dominate in a large number of habitats in invaded countries. In Japan, where $H$. axyridis is native, it is also a dominant predator of several species of aphid, but it coexists with various other aphidophagous predators with the guild displaying temporal and spatial niche differentiation (Osawa, 1991).

$H$. yedoensis had been regarded as a synonym of $H$. axyridis (e.g., Sasaji, 1971a), but was resurrected as a distinct species by Sasaji (1971b, 1977, 1981). Reproductive isolation between $H$. axyridis and $H$. yedoensis was found to be complete $(100 \%)$ when the two species mated under laboratory conditions (Okada et al., 1978; Sasaji, 1981). However, the ecology of $H$. yedoensis is poorly understood.

Harmonia axyridis and $H$. yedoensis are difficult to distinguish in the adult stage because of their morphological similarity. Adults of $H$. axyridis and $H$. yedoensis have four types of multi-colored elytra, and morphological differences between adults are observed only in the male genitalia (Sasaji, 1981; Nakagawa \& Sasaji, 1988). Furthermore, the ridge at the tip of the elytra (not all $H$. axyridis adults have the ridge on the elytra) is observed only in H. axyridis (Sasaji, 1981). Morphological differences at the larval stage, especially for third and forth instars, are distinct (Sasaji, 1977).

$H$. axyridis is regarded as a polyphagous predator, whereas $H$. yedoensis is thought to be an oligophagous aphid predator. The giant pine aphid Cinara pini Linne and Thunberg's pine aphid Eulachnus thunbergii Wilson are the only reported prey of $H$. yedoensis in field habitats (Tanigishi, 1975). The habitat of $H$. axyridis varies greatly (e.g., Osawa, 1993, 2000), whereas that of $H$. yedoensis is limited to pine trees (Tanigishi, 1975), where it can be found co-occuring with the more aggressive $H$. axyridis.

The habitats of $H$. axyridis are categorized into those suitable for survival and reproduction and those providing temporal refuge, according to density and quality of aphids in the habitats (Osawa, 2000). However, habitat segregation in $H$. yedoensis and $H$. axyridis is not based on strict difference in suitable food; at a laboratory condition, H. yedoensis develops normally, pupates, emerges, and oviposits when Cryptosiphum artemisiae Buckton and Macrosiphoniella sp. occur on Artemisia princeps (Sasaji, 1981) and Aphis spiraecola (Osawa, pers. observ.), which are prey for $H$. axyridis, but not for $H$. yedoensis in nature. These results suggest that ecological mechanisms may promote coexistence of the two sibling species on a pine tree, which is an interesting evolutionary issue. Furthermore, clarification of such mechanisms may aid in predicting how aphidophagous predators may interact with invasive populations of the aggressive $H$. axyridis in other parts of the world. In areas invaded by $H$. axyridis, aphidophagous predators are forced to cope with it, but potential mechanisms of coexistence remain to be determined.

We investigate possible mechanisms of coexistence of the sympatric sibling species, $H$. axyridis and $H$. yedoensis. We especially focus on the differences in their maternal investment through eggs and the role of sibling cannibalism at laboratory experiments.

\section{MATERIAL AND METHODS}

\section{Laboratory experiments}

The laboratory experiment was conducted from 27 May to 22 November, 2005. More than $100 \mathrm{H}$. axyridis pupae on Prunus persica infested by Myzus varians Davidson and on Salix koriyanagi infested by Chaitophorus horii Takahashi and eight adults of $H$. yedoensis (five females and three males) on Pinus densiflora (forma umbraculifera) infested by Cinara pini Linne were collected at the Botanical Garden of Kyoto University $\left(35^{\circ} 02^{\prime} \mathrm{N}\right.$ $135^{\circ} 47^{\prime} \mathrm{W}$ ) in mid May 2005. Forty newly-emerged adults of $H$. axyridis (20 females and 20 males) were randomly chosen before mating (ca. within a week after emergence) and used in the experiment. The eight $H$. yedoensis adults were held together as a stock culture with random mating. From them, we obtained many eggs and reared these offspring at each stage in plastic cups $(13 \mathrm{~cm}$ wide, $10 \mathrm{~cm}$ high) to the adult stage to obtain more than 100 newly-emerged adults. We provided the $H$. yedoensis larvae with a surplus of frozen Ephestia kuehniella Zeller eggs (Beneficial Insectary ${ }^{\circledR}$ ), which was changed daily and reared them at $25^{\circ} \mathrm{C}, 16 \mathrm{~L}: 8 \mathrm{D}$, and ca. $70 \%$ relative humidity. Of this second generation adults, 40 newly-emerged and unmated individuals (20 females and 20 males) were randomly chosen for the experiment. Newly-emerged adults of this second generation of $H$. yedoensis were used because (1) there were not enough unmated adults in the original field collection for the statistical analysis and (2) we could confirm the identification of H. yedoensis through larval morphology at the fourth instar.

We provided $H$. axyridis and $H$. yedoensis adults with the surplus of frozen E. kuehniella eggs, which was changed daily. To evaluate oviposition ability of females of $H$. axyridis and $H$. yedoensis without sperm shortage, the 20 experimental females of each species, each with a conspecific male, were individually held in plastic Petri dishes ( $7 \mathrm{~cm}$ wide, $2 \mathrm{~cm}$ high) at $25^{\circ} \mathrm{C}, 16 \mathrm{~L}$ $: 8 \mathrm{D}$, and ca. $70 \%$ relative humidity. They were provided with a surplus of frozen eggs, which was changed daily. Each day, we checked the Petri dishes, counted all eggs that were laid, and recorded the number of eggs per cluster. After removing the beetles, each egg cluster was individually kept in a labelled Petri dish at the same laboratory conditions and checked once or twice daily. All the females of $H$. axyridis and H. yedoensis were rared until they died.

In cases of sibling cannibalism in aphidophagous ladybird beetles, cannibals eat two types of sibling eggs in cluster form, undeveloped eggs and developed eggs with delayed hatching (Kawai, 1978; Osawa, 1992), although infertilization of the undeveloped eggs has not been confirmed. A cannibalized egg containing an embryo (the color is normally dark yellow when 


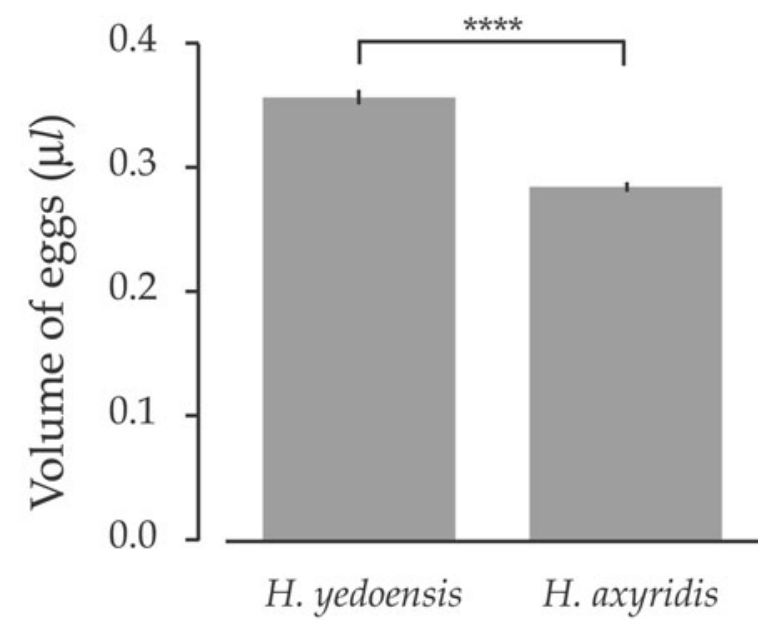

Fig. 1. Egg size in Harmonia yedoensis and H. axyridis. Vertical lines indicate S.E.

other eggs in the cluster hatch) is regarded as a developed egg with delayed hatching; that without an embryo (the color is normally light yellow when other eggs in the cluster hatch) is regarded as an undeveloped egg (Brown, 1972; Osawa, 1992). We distinguished the two types of cannibalized eggs under a stereo microscope (Carl Zeiss ${ }^{\circledR}$ SV 11 Apo) before, during, and after hatching. Hatching time of the cluster was regarded as the period from oviposition to the time of occurrence of the first larval hatching in a cluster.

We recorded the number of larvae $24 \mathrm{~h}$ after the eggs hatched. Thereafter, missing eggs were considered to have been cannibalized. In total, 103 egg clusters derived from five adults of $H$. yedoensis (i.e., no oviposition for 15 females) and 131 egg clusters derived from ten adults of $H$. axyridis (i.e., no oviposition for 10 females) were obtained until the females died and were used in the following analysis.

\section{Measurement of egg volume and cost}

To measure egg volume, another sets of 20 females and 23 males of $H$. axyridis at the first generation derived from the field population and 10 females and 13 males of $H$. yedoensis at the second generation derived from the eight field collected adults were placed together at the each stock cultures. They were rared with a surplus of frozen eggs in plastic cups $(13 \mathrm{~cm}$ wide, $10 \mathrm{~cm}$ high) at $25^{\circ} \mathrm{C}, 16 \mathrm{~L}: 8 \mathrm{D}$, and ca. $70 \%$ relative humidity. Ten eggs of $H$. yedoensis and 20 eggs of $H$. axyridis were randomly chosen from different egg batches of each stock culture, and egg length $(L)$ and width $(W)$ were measured to the nearest 0.025 $\mathrm{mm}$ using a micrometer with the stereo microscope.

Egg volume $(V, \mu \mathrm{l})$, which is regarded as the cost of egg production to the female, was calculated as: $V=L W^{2} \pi / 6$ (see Takakura, 2004). Using this value, oviposition cost was calculated as: Ovipositon cost $(\mu 1)=$ Egg volume $(V, \mu 1) \times$ Egg number. From this equation, the following were calculated: total oviposition cost per female throughout her lifetime, the cost of sibling cannibalism per cluster, the cost of undeveloped eggs per cluster, the cost of developed eggs with delayed hatching per cluster, and sibling cannibalism cost/larva per cluster .

\section{Statistical analysis}

Egg sizes of $H$. yedoensis and $H$. axyridis were compared by $t$-test. Similarly, $t$-tests were performed to compare $H$. yedoensis and $H$. axyridis for the following: cluster size, hatching time, number of clusters per female, total oviposition cost per female, percentage of hatching, number of undeveloped and developed
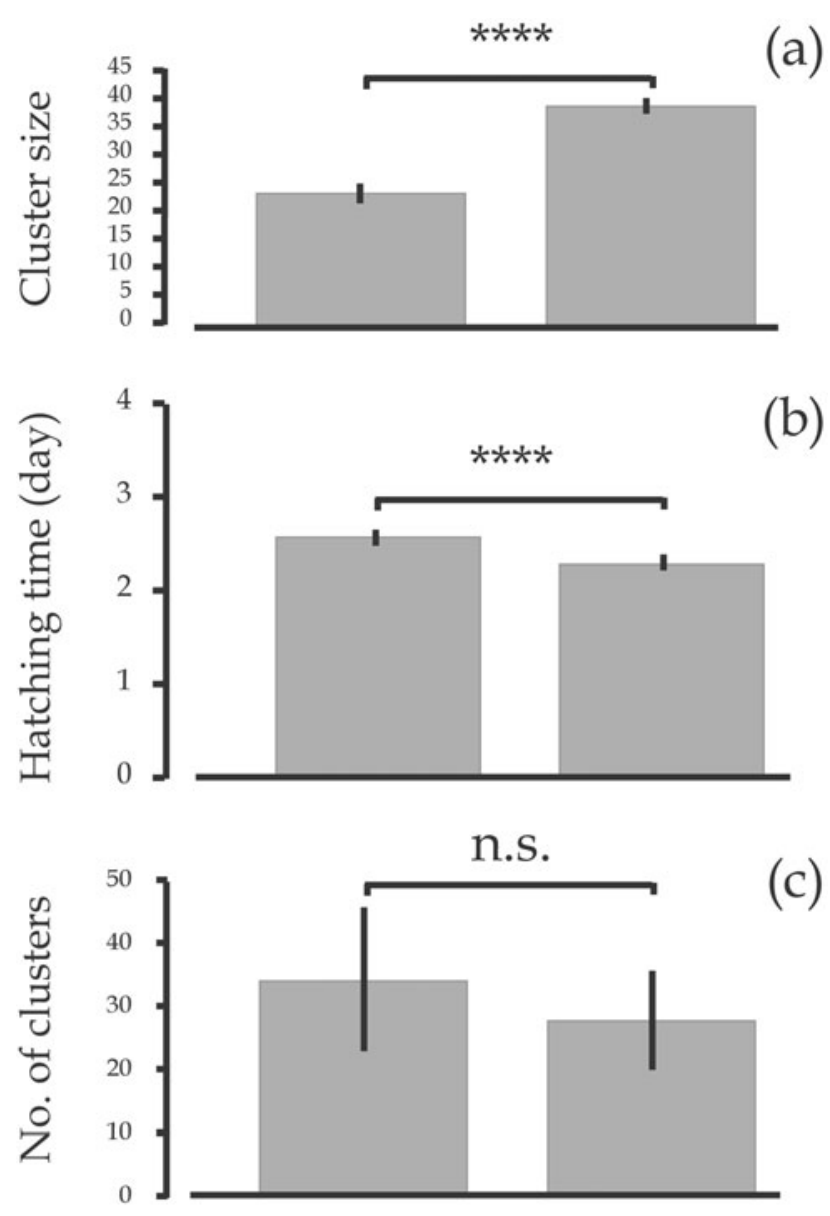

(c)

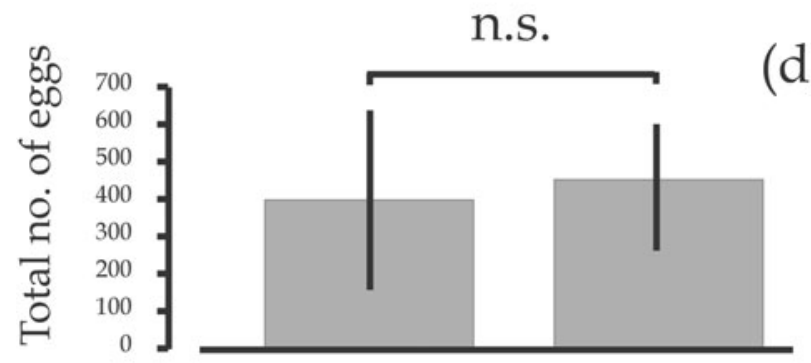

(d)

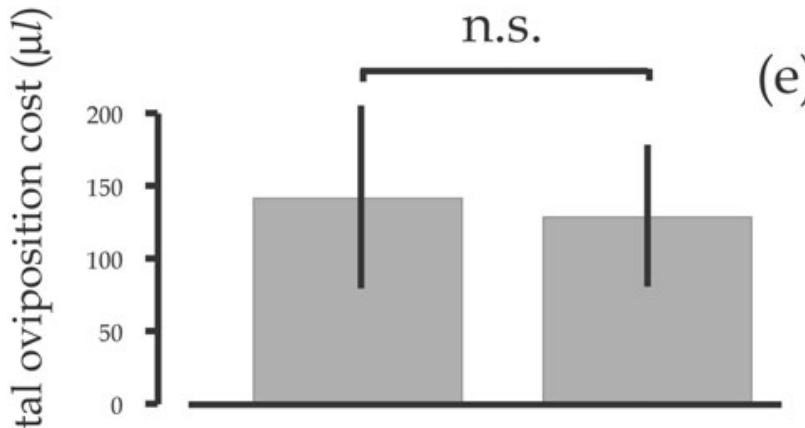

\section{H. yedoensis $H$. axyridis}

Fig. 2. Cluster size (a), hatching time (days) (b), number of clusters (c), total number of eggs (d), and total oviposition cost $(\mu l)(\mathrm{e})$ in Harmonia yedoensis and H. axyridis. Vertical lines indicate S.E. 


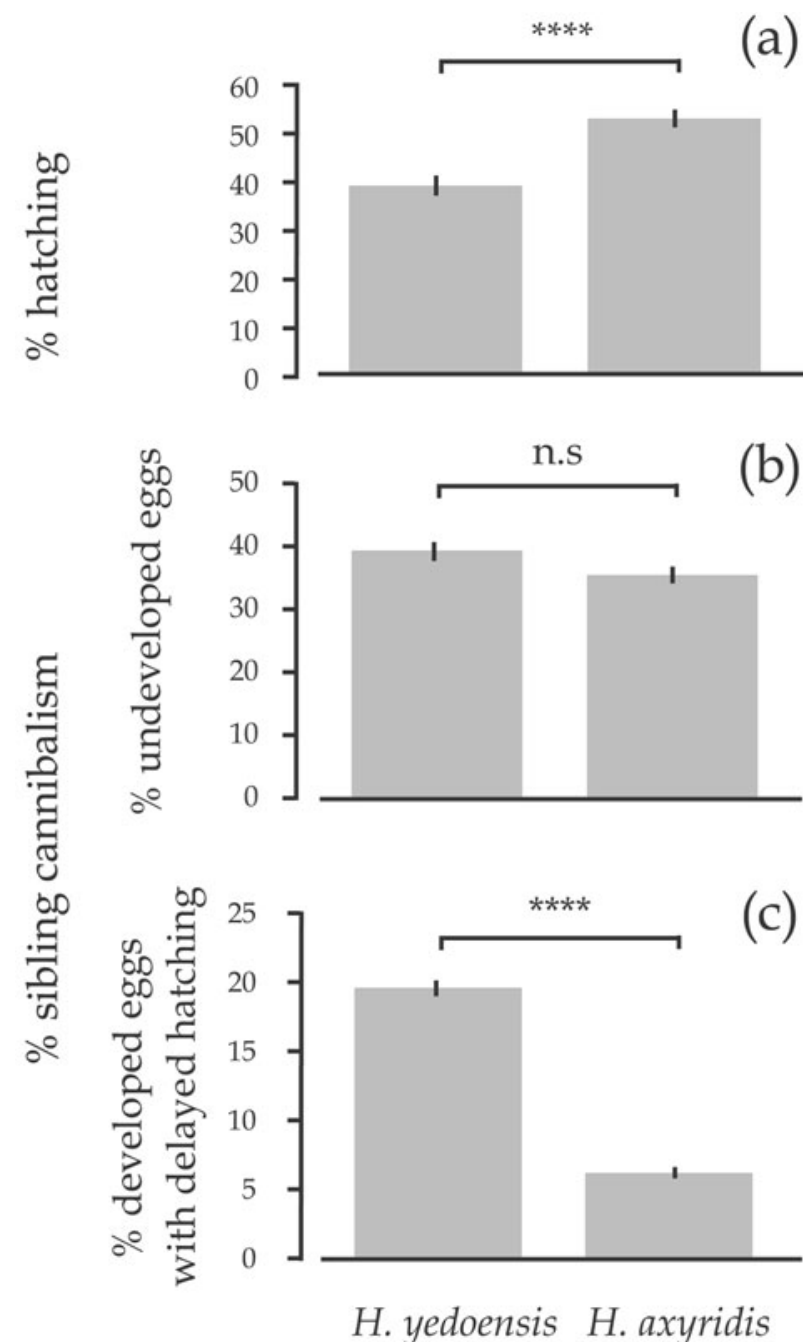

Fig. 3. The percentage of hatching (a), of undeveloped eggs (b), and of developed eggs with delayed hatching (c) per cluster in Harmonia yedoensis and H. axyridis. Vertical lines indicate S.E.

eggs with delayed hatching per cluster, and costs per cluster of oviposition, undeveloped eggs, developed eggs with delayed hatching per cluster, and sibling cannibalism/larva. We used a two-level nested ANOVA to analyze the relationship between the percentage of hatching, undeveloped eggs, and developed eggs with delayed hatching within cluster and (a) the size of that cluster, or (b) female age, for $H$. yedoensis and $H$. axyridis, respectively. We eliminated one cluster from this analysis because one female produced only that cluster and thereafter we could not evaluate the female effect of this cluster. In total, 103 clusters from five females of $H$. yedoensis and 130 clusters from nine females of $H$. axyridis were used in this analysis. All of the percentage data were arcsine transformed. We used JMP Discovery Software (SAS, 2000) for all statistical analyses.

\section{RESULTS}

\section{Characteristics of eggs and sibling cannibalism}

Female size in $H$. yedoensis $(7.0985 \pm 0.0729 \mathrm{~mm}, \mathrm{n}=$ 20) was not significantly different from that in $H$. axyridis $(7.2555 \pm 0.0726 \mathrm{~mm}, \mathrm{n}=20)(t$-test, $t=1.5238, p=$ 0.1358). The volume of Harmonia yedoensis eggs $(0.3564 \pm 0.0094 \mu \mathrm{l}$, mean \pm S.E. $)$ was $24.91 \%$ larger
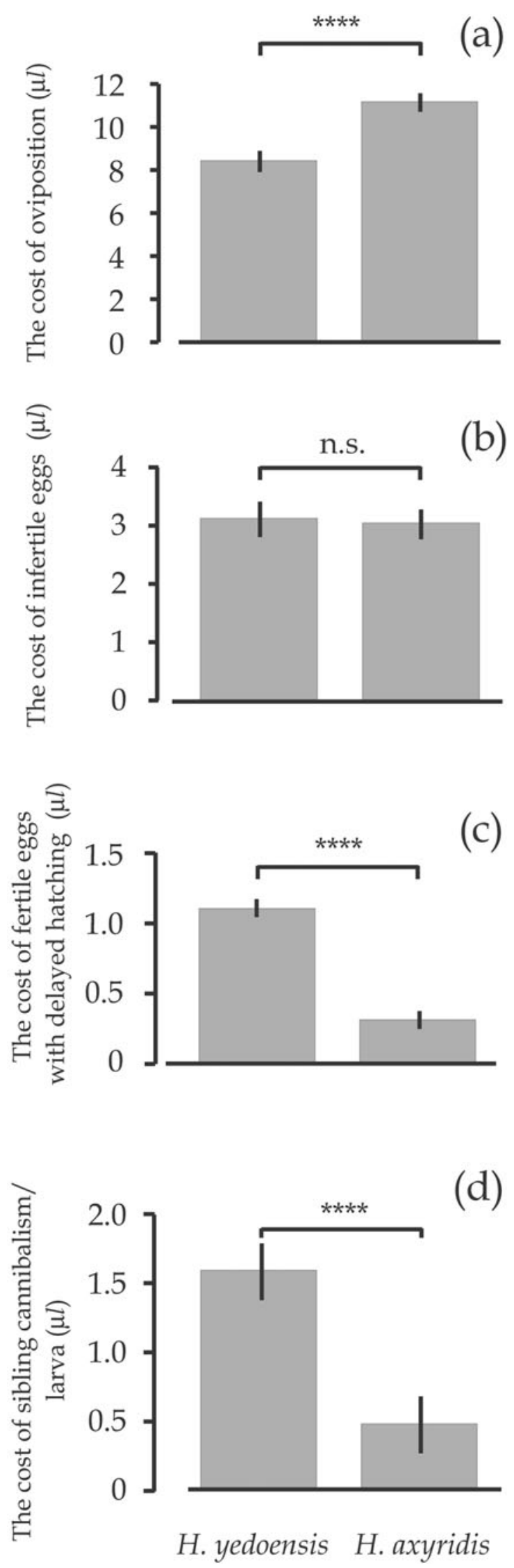

Fig. 4. The cost of oviposition $(\mu \mathrm{l})(\mathrm{a})$, of undeveloped eggs $(\mu l)(b)$, of developed eggs with delayed hatching $(\mu l)(c)$, and of sibling cannibalism/larva (d) per cluster in Harmonia yedoensis and $H$. axyridis. Vertical lines indicate S.E. 
(a)

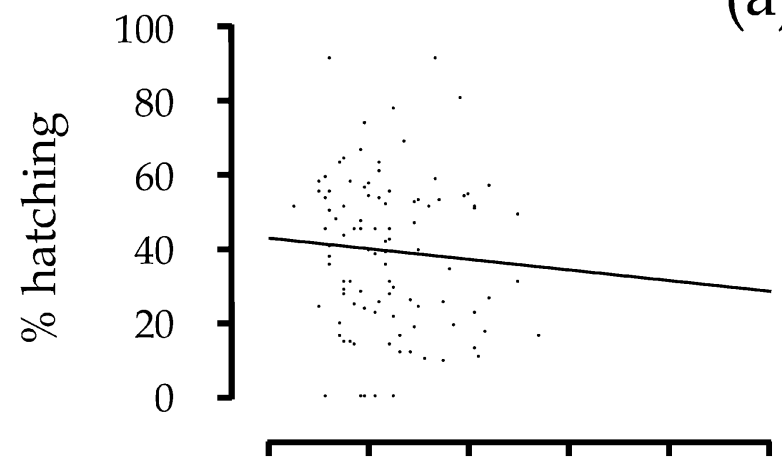

(b)

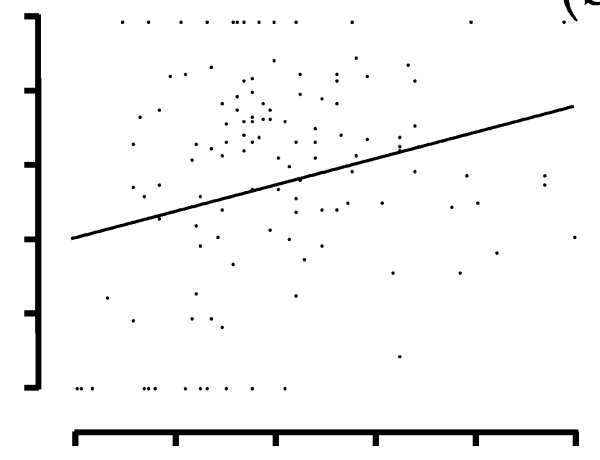

(d)
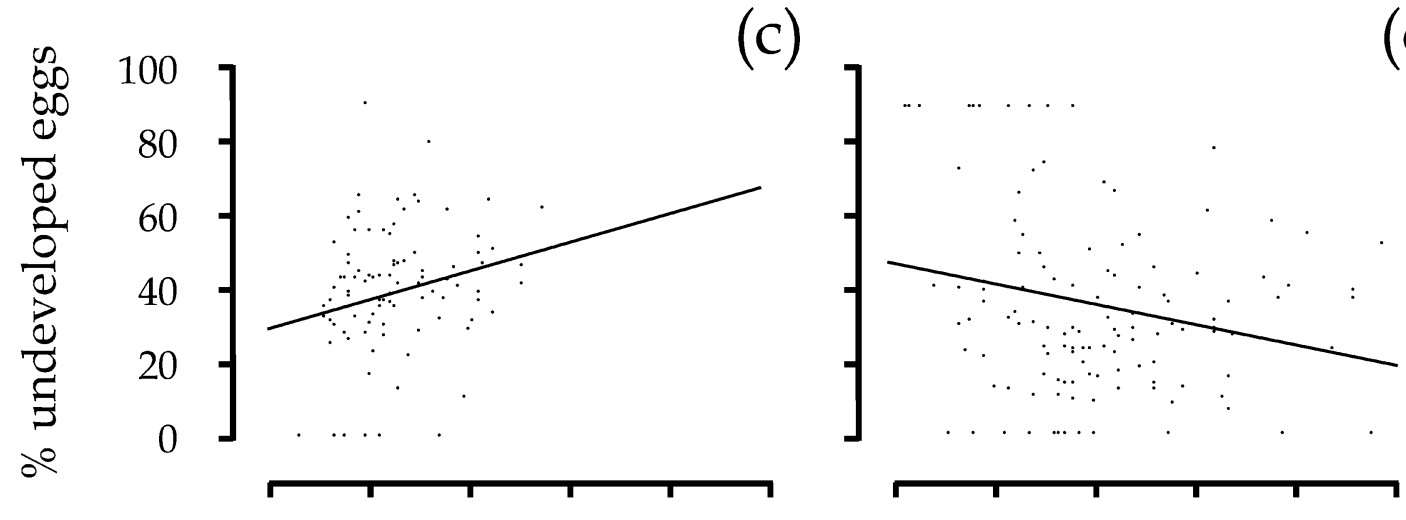

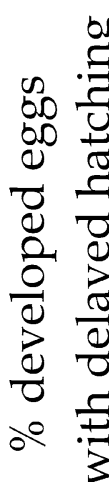
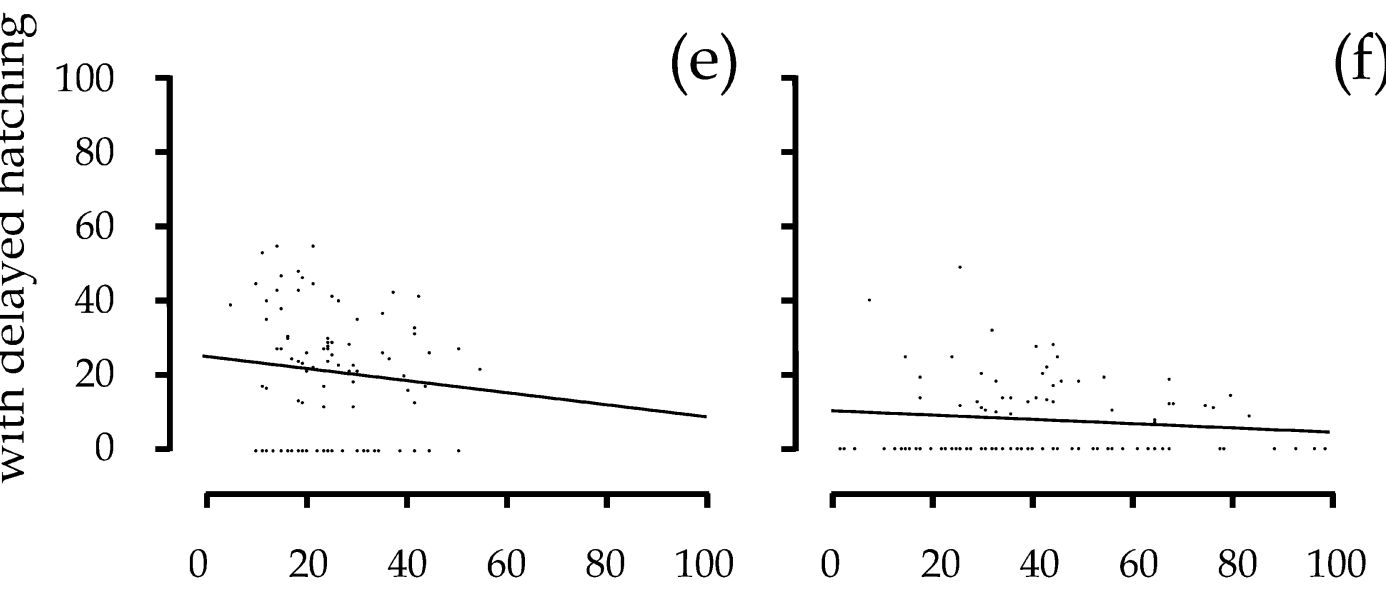

\section{Cluster size}

Fig. 5. The relationship between cluster size and percentage hatching (a, b), that between cluster size and percentage of undeveloped eggs (c, d), and that between cluster size and percentage of developed eggs with delayed hatching (e, f) per cluster in Harmonia yedoensis and $H$. axyridis, respectively.

than that of $H$. axyridis $(0.2848 \pm 0.0067)$ ( $t$-test: $t=$ $6.2054, p<0.0001$; Fig. 1). Cluster size of $H$. axyridis $(39.1069 \pm 1.4742$, mean \pm S.E. $)$ was significantly larger than that of $H$. yedoensis $(23.5631 \pm 1.6626)(t$-test: $t=$ 6.9952, $p<0.0001$; Fig. 2a). Hatching time of $H$. yedoensis (3.0106 \pm 0.0584 day) was significantly longer than that of $H$. axyridis $(2.6754 \pm 0.0530)(t$-test: $t=$ $4.2512, p<0.0001$; Fig. 2b). The number of clusters $(20.6000 \pm 6.4903)$, total number of eggs $(401.2000 \pm$ 231.1600), and total oviposition cost per female $(142.9700 \pm 68.3360 \mu \mathrm{l})$ in $H$. yedoensis were not significantly different from those in $H$. axyridis $(13.1000 \pm$ 
H. yedoensis

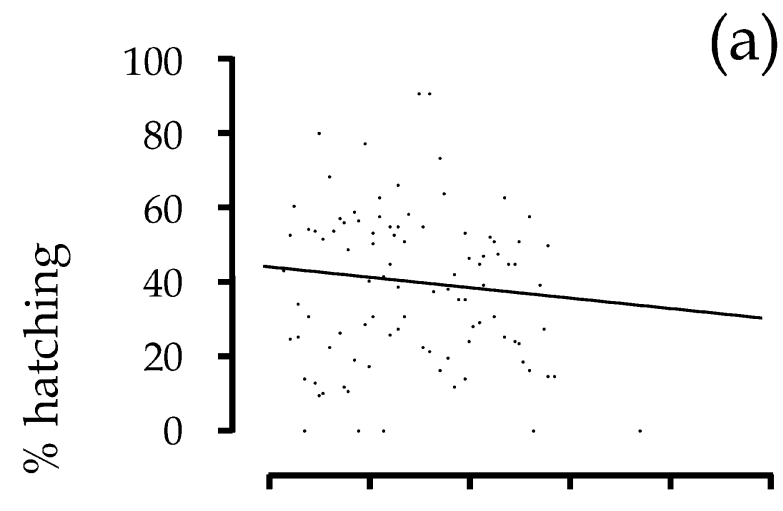

(a)

\section{H. axyridis}

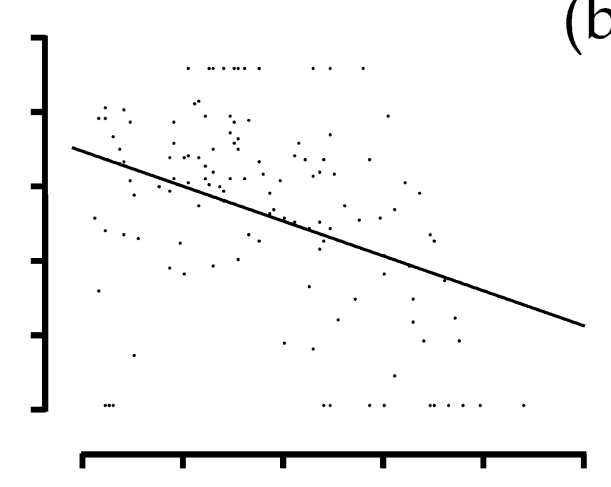

(b)

(d)

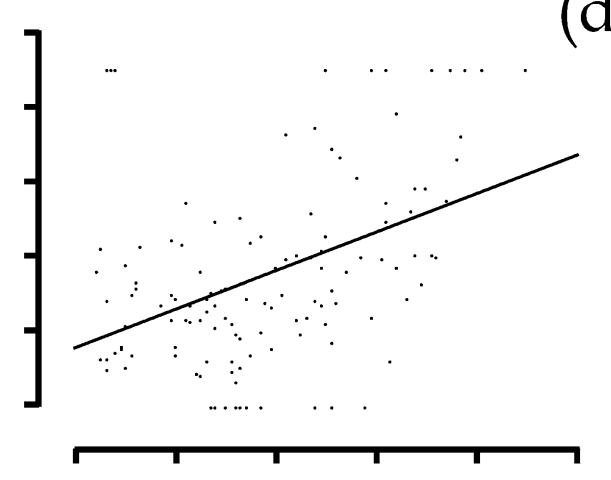

(f)

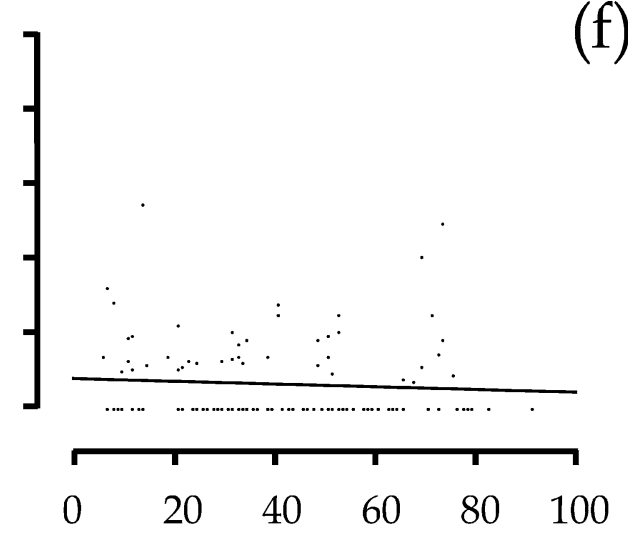

Age (day)

Fig. 6. The relationship between female age and percentage hatching $(a, b)$, that between female age and percentage of undeveloped eggs (c, d), and that between female age and percentage of developed eggs with delayed hatching (e, f) per cluster in Harmonia yedoensis and $H$. axyridis, respectively.

$4.5894,455.4000 \pm 163.4600$, and $129.6960 \pm 48.3210$, respectively) ( $t$-test: $t=0.9435, p=0.3626 ; t=0.1914, p$ $=0.8511$; and $t=0.1586, p=0.8764$, respectively), although total number of clusters tended to be slightly greater in H. yedoensis than in H. axyridis (Fig. 2c-e).
The percentage of hatching per cluster in $H$. axyridis $(51.9395 \pm 2.1220 \%$, mean \pm S.E. $)$ was significantly higher than that in $H$. yedoensis $(38.2994 \pm 2.4034 \%)$ ( $t$-test: $t=4.2544, p<0.0001$; Fig. 3a). In sibling cannibalism, the percentage of undeveloped eggs per cluster in H. yedoensis $(39.1838 \pm 2.2220 \%)$ was not significantly 
different from that in $H$. axyridis $(35.4255 \pm 1.9596 \%)$ ( $t$-test: $t=1.2685, p=0.2059$; Fig. $3 \mathrm{~b}$ ), whereas the percentage of developed eggs per cluster with delayed hatching in $H$. yedoensis $(19.5105 \pm 1.3735 \%)$ was significantly greater than that in $H$. axyridis $(6.0382 \pm$ $1.2127 \%$ ) ( $t$-test: $t=7.3531, p<0.0001$; Fig. $3 \mathrm{c})$.

The cost of oviposition per cluster in $H$. axyridis $(11.1374 \pm 0.4400 \mu \mathrm{l}$, mean \pm S.E. $)$ was $32.64 \%$ larger than that in $H$. yedoensis $(8.3968 \pm 0.4962)$, a significant difference ( $t$-test: $t=4.1328, p<0.001$; Fig. 4a). In cases of sibling cannibalism, the cost of undeveloped eggs per cluster in $H$. yedoensis $(3.1449 \pm 0.3167 \mu \mathrm{l})$ was not significantly different from that in $H$. axyridis (3.0654 \pm $0.2808 \mu \mathrm{l})(t$-test: $t=3.9186, p=0.8510$; Fig. 4b), whereas the cost per cluster of developed eggs with delayed hatching in $H$. yedoensis $(1.1071 \pm 0.0976 \mu \mathrm{l})$ was 3.49 times greater than that in $H$. axyridis $(0.3174 \pm$ $0.0865 \mu \mathrm{l})(t$-test: $t=6.0541, p<0.0001$; Fig. 4c). Furthermore, the cost of sibling cannibalism per hatched larva/cluster in $H$. yedoensis $(1.5781 \pm 0.2094 \mu \mathrm{l})$ was 3.36 times greater than that in $H$. axyridis $(0.4696 \pm$ $0.1902 \mu \mathrm{l})$, and the difference was statistically significant ( $t$-test: $t=3.9186, p<0.0001$; Fig. $4 \mathrm{~d}$ ).

\section{Factors affecting sibling cannibalism}

Cluster size was not a significant factor affecting the percentage of hatching per cluster in H. yedoensis (Twolevel nested ANOVA: female code, $F=21.3869, d f=4, p$ $<0.0001$; cluster size (female code) $F=0.7306, d f=5, p$ $=0.6023$ ) (whole model: $\mathrm{R}^{2}=0.5534, F_{9,89}=12.2541, p<$ $0.0001)$, but it was a significant factor in $H$. axyridis (female code, $F=2.1696, d f=8, p=0.0353$; cluster size [female code]; $F=2.0558, d f=9, p=0.0398$ ) (whole model: $\mathrm{R}^{2}=0.2793, F_{17,109}=2.4851, p=0.0024$; Fig. 5a, b). In sibling cannibalism, cluster size was a marginally significant factor affecting the percentage of undeveloped eggs per cluster in $H$. yedoensis (female code, $F=6.0952$, $d f=4, p=0.0002$; cluster size [female code]; $F=2.0733$, $d f=5, p=0.0763$ ) (whole model: $\mathrm{R}^{2}=0.3600, F_{9,88}=$ 5.4992, $p<0.0001$ ), and a significant factor in $H$. axyridis (female code, $d f=8, F=1.9284, p=0.0629$; cluster size [female code]; $F=1.9730, d f=9, p=0.0493$ ) (whole model: $\mathrm{R}^{2}=0.2677, F_{17,108}=2.3229, p=0.0047$; Fig. 5c, d). However, cluster size did not significantly affect the percentage of developed eggs with delayed hatching per cluster in either $H$. yedoensis (female code, $F=20.1566, d f=4, p<0.0001$; cluster size [female code]; $F=1.2607, d f=5, p=0.2880$ ) (whole model: $\mathrm{R}^{2}=$ $\left.0.5573, F_{9,89}=12.4473, p<0.0001\right)$ or $H$. axyridis (female code, $F=4.5698, d f=8, p<0.0001$; cluster size [female code]; $F=1.0529, d f=9, p=0.4035$ ) (whole model: $\mathrm{R}^{2}=0.3305, F_{17,109}=3.1654, p<0.0001$; Fig. 5e, f).

Female age significantly influenced the hatching percentage per cluster in both $H$. yedoensis (two-level nested ANOVA: female code, $F=8.6974, d f=4, p<0.0001$; cluster size [female code]; $F=2.6354, d f=5, p=0.0286$ ) (whole model: $\mathrm{R}^{2}=0.5950, F_{9,89}=14.5302, p<0.0001$ ) and $H$. axyridis (female code, $F=5.2359, d f=8, p<$ 0.0001 ; cluster size [female code]; $F=8.6565, d f=9, p<$
0.0001) (whole model: $\mathrm{R}^{2}=05084, F_{17,109}=6.6303, p<$ 0.0001; Fig. 6a, b). In sibling cannibalism, female age was not a significant factor affecting the percentage of undeveloped eggs per cluster in $H$. yedoensis (female code, $F=2.0823, d f=4, p=0.0899$; cluster size [female code]; $F=0.7725, d f=5, p=0.5721$ ) (whole model: $\mathrm{R}^{2}=$ $\left.0.3147, F_{9,88}=4.4891, p<0.0001\right)$, but it was a significant factor in $H$. axyridis (female code, $F=5.1211, d f=$ $8, p<0.0001$; cluster size [female code]; $F=7.9024, d f=$ $9, p<0.0001$ ) (whole model: $\mathrm{R}^{2}=0.4859, F_{17,108}=$ $6.0045, p<0.0001$; Fig. $6 \mathrm{c}, \mathrm{d})$. Female age did not significantly affect the percentage of developed eggs with delayed hatching per cluster in either $H$. yedoensis (female code, $F=15.9044, d f=4, p<0.0001$; cluster size [female code]; $F=1.8479, d f=5, p=0.1116$ ) (whole model: $\left.\mathrm{R}^{2}=0.5705, F_{9,89}=13.1354, p<0.0001\right)$ or $H$. axyridis (female code, $F=1.1796, d f=8, p=0.3179$; cluster size [female code]; $F=1.2298, d f=9, p=0.2844$ ) (whole model: $\mathrm{R}^{2}=0.3394, F_{17,109}=3.2941, p<0.0001$; Fig. 6e, f).

\section{DISCUSSION}

Egg size can affect progeny fitness; larger offspring often mature earlier, to have improved ability to avoid or withstand predation and competition, or to have greater survival in stressful environments in comparison with smaller offspring (e.g., Azevedo et al., 1997; Fox \& Czesak, 2000; Roff, 2002; Czesak \& Fox, 2003; Fischer et al., 2003, 2006, 2007). Conversely, a female cannot increase offspring number without decreasing the size of individuals because of the presumed trade-off between number and size of offspring, which is a fundamental principle of life history theory in many taxa (e.g., Vance, 1973; Smith \& Fretwell, 1974; Brockelman, 1975; McGinley et al., 1987; Sinervo 1990; Sterns, 1992; Roff, 2002). This study showed that: (1) cluster size in Harmonia axyridis was significantly larger but egg size was smaller than that in $H$. yedoensis, (2) the number of $H$. yedoensis clusters tended to be slightly higher than that of $H$. axyridis, and (3) total number of eggs and oviposition cost per female in $H$. yedoensis over her life time were not significantly different from those in $H$. axyridis. The maternal investment of the two species differed; $H$. yedoensis females lay larger eggs in fewer numbers, whereas $H$. axyridis females lay smaller eggs in larger numbers. The end result is almost equal reproductive effort between $H$. yedoensis and $H$. axyridis. Furthermore, female size is generally thought to determine the number of progeny (e.g., Thornhill \& Alcock, 1983; Birkhead et al., 1998). Female size in $H$. yedoensis was not significantly different from that in $H$. axyridis in this study, implying further support to the equality of reproductive effort between $H$. yedoensis and $H$. axyridis.

Egg size reflects maternal reproductive investment and the initial amount of resources available to progeny (e.g., Sinervo, 1990). In $H$. yedoensis and $H$. axyridis, mothers do not care for offspring; thus, egg size has an especially important role in determining maternal investment in these species. Several characteristics of these two species, 
including morphology in adult and larval stages, are difficult to distinguish and they overlap in habitat on pine trees (e.g., Sasaji, 1977). However, this study showed that maternal investment in offspring, as measured the volume of eggs, was $24.91 \%$ greater in $H$. yedoensis than in $H$. axyridis (Fig. 1). These results suggest that larval survival rate, especially in the first instar, may be higher in $H$. yedoensis than in $H$. axyridis under natural conditions because of higher ability of prey capturing based on larger maternal investment in eggs.

A strong positive relationship exists between reproductive biomass and adult weight, and egg size is a function of adult weight and ovariole number in coccinellids (Stewart et al., 1991). Furthermore, Dixon \& Guo (1993) predicted that egg size is the least variable reproductive trait and that cluster size is the most variable. These results suggest that maternal investment in eggs is allometrically determined by adult weight and ovariole number. In general, egg size is constrained by the minimum size at which first instar larvae can capture active prey and complete their development before prey become scarce (Stewart et al., 1991). However, food conditions around newly hatched first instar larvae are critical, mainly because of the difficulties larvae experience in capturing prey (Dixon, 1959; Kawai, 1978) and the fact that the oviposition site is set apart from aphid colonies to avoid non-sibling cannibalism in $H$. axyridis (Osawa, 1989). Thus, maternal investment through egg size alone may be insufficient for newly hatched $H$. yedoensis and $H$. axyridis larvae to develop into adults. The cost of sibling cannibalism per hatched larva/cluster in $H$. yedoensis was 3.36 times greater than that in $H$. axyridis (Fig. 4d), suggesting that the realized maternal investment for offspring in $H$. yedoensis is actually more than 3.36 times that in $H$. axyridis when combined with investment through an egg.

The maternal investment in each $H$. yedoensis larva (1.5781 $\pm 0.2094 \mu \mathrm{l}$ in Fig. 4 d) amounts to 4.43 eggs (egg volume in $H$. yedoensis: $0.3564 \pm 0.0094 \mu \mathrm{l}$ inFig. 1$)$, when egg cannibalism is included. In $H$. axyridis, the survival rate of first instar larvae increases 6.27 times when larvae cannibalize three eggs at low aphid densities and 2.58 times at high aphid densities (Osawa, 1992). Furthermore, newly hatched $H$. axyridis larvae successfully molt to the second instar when they feed on three eggs (Kawai, 1978; Osawa, 1991). Assuming that the beneficial effect of sibling cannibalism on the first instar is similar in $H$. yedoensis, the greater initial larval size of $H$. yedoensis, combined with the maternal investment through sibling cannibalism at hatching, may further improve the survival of the first instar $H$. yedoensis relative to the first instar $H$. axyridis. Thus, the actual maternal investment per offspring is 4.197[1.249 (in egg size) $\times 3.36$ (in sibling cannibalism)] times greater in $H$. yedoensis than in $H$. axyridis when the maternal investments in egg size and sibling cannibalism are combined.

Sibling cannibalism promotes more rapid development and larger adult size in $H$. axyridis, suggesting that faster development may be adaptive for resource tracking, and large adult size increases fecundity in females and mating success in males through female mate choice, both resulting in an increase in the fitness of cannibals (Osawa, 2002). Furthermore, this study showed that hatching time of egg clusters was longer in H. yedoensis than in H. axyridis (Fig. 2b). A positive relationship between egg size and hatching time is evident in Diptera and Coleoptera (Azevedo et al., 1997; Fox, 1997), suggesting that eggs of $H$. yedoensis develop more slowly than those of $H$. axyridis. Therefore, the effectiveness of maternal investment through sibling cannibalism in increasing adult body size and shortening developmental period may be superior to that through eggs, which may be an ultimate factor favoring maternal investment through sibling cannibalism.

In sibling cannibalism in $H$. axyridis, a cannibal can choose from two types of eggs: undeveloped eggs or developed eggs with delayed hatching (Osawa, 1992). These two types of eggs exist in both $H$. axyridis and $H$. yedoensis. Undeveloped/trophic eggs are regarded as a direct maternal investment (i.e., the mother can directly control their production) in offspring (Osawa, 2003), although the infertility of the undeveloped egg and the control mechanisms for the production of the undeveloped eggs have yet to be clarified. The undeveloped eggs are regarded to be a maternal investment in $H$. axyridis; the proportion of undeveloped/trophic eggs in a cluster is manipulated based on information from prey encounters (Perry \& Roitberg, 2005). The present study showed that cluster size as well as the identity of the mother significantly affected the percentage of undeveloped eggs per cluster in $H$. axyridis, and cluster size was marginally significant in $H$. yedoensis (Fig. 5). Furthermore, age of mothers was a significant factor affecting the percentage of undeveloped eggs per cluster in $H$. axyridis, the reverse was true in $H$. yedoensis (Fig. 6). These results suggest that physiological mechanisms for the production of eggs may be combined with maternal manipulation in the production of undeveloped eggs in $H$. yedoensis and H. axyridis.

In $H$. axyridis, Osawa (1992) explained sibling cannibalism of developed eggs with delayed hatching through kin selection with three potential beneficiaries, i.e., the cannibal, the victim, and the mother. The altruistic behavior of being a victim was beneficial to the victim only when the victim was cannibalized by full siblings at low aphid densities. At the same time, cannibalism was not maladaptive for the mother; mothers attained almost equal fitness regardless of the proportion of developed eggs with delayed hatching. Therefore, intense kin relatedness between the cannibal and the victim, and low survival rate of the siblings in cluster favor the occurrence of sibling cannibalism.

The proximate factor for occurrence of sibling cannibalism was hatching asynchrony in clusters (Osawa, 1992). Male-killing Spiroplasma plays a role in the production of developed eggs with delayed hatching (Nakamura et al., 2006); however, the precise mechanism is not fully understood. In birds, asynchronous hatching is gen- 
erally believed to result from the asynchronous timing of egg development (e.g., Ricklefs, 1993). Cluster size therefore may be involved in the occurrence of sibling cannibalism, if variation in developmental time arises from eggs being laid over an extended period of time within a cluster. However, our study showed that cluster size-related mechanisms are not involved in the production of developed eggs with delayed hatching in $H$. yedoensis and $H$. axyridis, implying that independently of cluster size, the female of $H$. yedoensis and $H$. axyridis manipulates the hatching time of offspring in a cluster. Parental strategies exist to alleviate the effects of asynchronous hatching in clusters (e.g., in birds: Wiebe \& Bortolotti, 1994; Much \& Nager, 2006; in insects: Frechette \& Coderre, 2000). The implication of this study, i.e., mothers of $H$. yedoensis and $H$. axyridis may manipulate the hatching time of their offspring, suggests that the production of developed eggs with delayed hatching is a secondary mechanism for manipulating sibling cannibalism opportunities.

This study showed that the percentage and cost of developed eggs with delayed hatching per cluster was greater for $H$. yedoensis than for $H$. axyridis, whereas the percentage and cost of undeveloped eggs per cluster was not significantly different between the two species. These results suggest that sibling cannibalism of developed eggs with delayed hatching is most important in determining the contrasting intensity of cannibalism between $H$. yedoensis and $H$. axyridis. One notes that the intensity of sibling cannibalism of developed eggs with delayed hatching does not affect the mother's fitness regardless of food conditions for offspring, but the intensity of sibling cannibalism of undeveloped eggs reduces the mother's fitness at high aphid densities for the offspring in $H$. axyridis (Osawa, 1992). Thus, maternal investment in sibling cannibalism of developed eggs with delayed hatching may be an especially adaptive reproductive strategy that does not reduce the mother's fitness also in $H$. yedoensis.

ACKNOWLEDGEMENTS.We are indebted to H. Takeda, Kyoto University, for his support, suggestions, and encouragement during this study. This study was supported in part by a Grant-in-Aid for Science Research (No. 20405047, to N. Osawa) and the COE for Innovative Food and Environmental Studies Pioneered by Entomomimetic Sciences (the Ministry of Education, Culture, Sports, Science, and Technology of Japan).

\section{REFERENCES}

Adriaens T., Branquart E. \& Maes D. 2003: The multicoloured Asian ladybird Harmonia axyridis Pallas (Coleoptera: Coccinellidae), a threat for native aphid predators in Belgium? Belg. J. Zool. 133: 207-208.

Azevedo R.B.R., French V. \& Partridge L. 1997: Life-history consequences of egg size in Drosophila melanogaster. Am. Nat. 150: 250-282.

BirkheAd T.R., LeE K.E. \& Young P. 1998: Sexual cannibalism in the praying mantis Hierodula membranacea. Behavior 106: $112-118$.

Brockelman W.Y. 1975: Competition, the fitness of offspring, and optimal clutch size. Am. Nat. 109: 677-399.
BRown H.D. 1972: The behaviour of newly hatched coccinellid larvae (Coleoptera: Coccinellidae). J. Entomol. Soc. Sth Afr. 35: 149-157.

BRown M.W. 2003: Intraguild responses of aphid predators on apple to the invasion of an exotic species, Harmonia axyridis. BioControl 48: 141-153.

Colunga-García M. \& GAGE S.H. 1998: Arrival, establishment, and habitat use of the multicolored Asian lady beetle (Coleoptera: Coccinellidae) in a Michigan landscape. Environ. Entomol. 27: 1574-1580.

Czesak M.E. \& Fox C.W. 2003: Evolutionary ecology of egg size and number in a seed beetle: genetic trade-off differs between environments. Evolution 57: 1121-1132.

Dixon A.F.G. 1959: An experimental study of the searching behaviour of the predatory coccinellid beetle Adalia bipunctata (L.). J. Anim. Ecol. 28: 259-281.

Dixon A.F.G. \& Guo Y. 1993: Egg and cluster size in ladybird beetles (Coleoptera: Coccinellidae): The direct and indirect effects of aphid abundance. Eur. J. Entomol. 90: 457-463.

Fischer K., Bot A.N.M., Brakefield P.M. \& ZwaAn B.J. 2003: Fitness consequences of temperature-mediated egg size plasticity in a butterfly. Funct. Ecol. 17: 803-810.

Fischer K., Bot A.N.M., Brakefield P.M. \& ZwaAn B.J. 2006: Do mothers producing large offspring have to sacrifice fecundity? J. Evol. Biol. 19: 380-391.

Fischer K., ZwaAn B.J. \& Brakefield P.M. 2007: Realized correlated responses to artificial selection on pre-adult lifehistory traits in a butterfly. Heredity 98: 157-164.

Fox C.W. 1997: Egg size manipulations in the seed beetle, Stator limbatus: consequences for progeny growth. Can. J. Zool. 75: 1465-1473.

Fox C.W. \& CZEsaK M.E. 2000: Evolutionary ecology of progeny size in arthropods. Annu. Rev. Entomol. 45: 341-369.

Fréchette B. \& Coderre D. 2000: Oviposition strategy of the green lacewing Chrysoperla rufilabris (Neuroptera: Chrysopidae) in response to extraguild prey availability. Eur. J. Entomol. 97: 507-510.

HodeK I. \& HonĚK A. 1996: Ecology of Coccinellidae. Kluwer Academic Publishers, Dordrecht, 464 pp.

KaWAI A. 1978: Sibling cannibalism in the first instar larvae of Harmonia axyridis Pallas (Coleoptera: Coccinelidae). Konty $\hat{u}$ 46: 14-19.

Koch R.L. 2003: The multicolored Asian lady beetle, Harmonia axyridis: a review of its biology, uses in biological control, and non-target impacts. J. Insect Sci. 3: 32-47.

McGinley M.A., Temme D.H. \& Geber M.A. 1987: Parental investment in offspring in variable environments: theoretical and empirical considerations. Am. Nat. 130: 370-398.

Much C. \& Nager R.G. 2006: The effect of laying and hatching order on the timing and asynchrony of hatching. Anim. Behav. 71: 885-892.

Nakagawa K. \& SASAJI H. 1988: Elytral colour pattern polymorphisms and their inheritance in Harmonia yedoensis Takizawa (Coleoptera: Coccinellidae). Entomol. J. Fukui 2: 37-48 [in Japanese with English abstr.].

Nakamura K., Miura K., Jong P.D. \& Ueno H. 2006: Comparison of the incidence of sibling cannibalism between malekilling Spiroplasma infected and uninfected clutches of a predatory ladybird beetle, Harmonia axyridis (Coleoptera: Coccinellidae). Eur. J. Entomol. 103: 323-326.

OKada I., NiJima K. \& ToRiumi Y. 1978: Comparative studies on sibling species of lady beetles, Harmonia yedoensis and $\mathrm{H}$. axyridis (Coleoptera: Coccinellidae). Bull. Fac. Agr. Tamagawa Univ. 18: 60-68 [in Japanese with English abstr.]. 
Osawa N. 1989: Sibling and non-sibling cannibalism by larvae of a lady beetle Harmonia axyridis Pallas (Coleoptera: Coccinelidae). Res. Popul. Ecol. 31: 153-160.

Osawa N. 1991: Ecological Studies on the Ladybird Beetle Harmonia axyridis Pallas in a Natural Population. Ph. D. Thesis, Kyoto University, $184 \mathrm{pp}$. [in Japanese].

OsawA N. 1992: Sibling cannibalism in the ladybird beetle Harmonia axyridis Pallas: fitness consequences for mother and offspring. Res. Popul. Ecol. 34: 45-55.

Osawa N. 1993: Population field studies of the aphidophagous ladybird beetle Harmonia axyridis (Coleoptera: Coccinellidae): life tables and key factor analysis. Res. Popul. Ecol. 35: 335-348.

Osawa N. 2000: Population field studies on the aphidophagous ladybird beetle Harmonia axyridis (Coleoptera: Coccinellidae): resource tracking and population characteristics. Popul. Ecol. 42: 115-127.

Osawa N. 2002: Sex-dependent effects of sibling cannibalism on life history traits of the ladybird beetle Harmonia axyridis (Coleoptera: Coccinellidae). Biol. J. Linn. Soc. 76: 349-360.

OsawA N. 2003: The influence of female oviposition strategy on sibling cannibalism in the ladybird beetle Harmonia axyridis (Coleoptera: Coccinellidae). Eur. J. Entomol. 100: 43-48.

Perry J.C. \& RoItberg B.D. 2005: Ladybird mothers mitigate offspring starvation risk by laying trophic eggs. Behav. Ecol. Sociobiol. 58: 578-586.

RICKLEFS R.E. 1993: Sibling competition, hatching asynchrony, incubation period, and lifespan in altricial birds. In Power D.M. (ed.): Current Ornithology. Vol. 11. Plenum, New York, pp. 199-276.

RIDLEY M. 2004: Evolution. 3rd ed. Blackwell, Oxford, 751 pp.

Roff D.A. 2002: Life History Evolution. Sinauer Associates, Sunderland, MA, $465 \mathrm{pp}$.

SAS Institute 2000: JMP Statistical Discovery Software (ver. 4). SAS Institute Inc., Cary, NC.

SASAJ H. 1971a: Fauna Japonica Coccinellidae (Insecta: Coleoptera). Academic Press of Japan, Tokyo, 340 pp.

SASAJI H. 1971b: Two species of Harmonia axyridis auct. Paper presented at the $31^{\text {st }}$ Annual Meeting of the Entomol. Soc. Japan. [in Japanese].

SASAJI H. 1977: Larval characters of Asian species of the genus Harmonia Mulsant (Coleoptera: Coccinellidae). Mem. Fac. Educ. Fukui Univ. (Ser. II, Nat. Sci.) 27: 1-18.
SASAJI H. 1981: Biosystematics on Harmonia axyridis-complex (Coleoptera: Coccinellidae). Mem. Fac. Educ. Fukui Univ. (Ser. II. Nat. Sci.) 30: 59-79.

SinERvo B. 1990: The evolution of maternal investment in lizards: an experimental and comparative analysis of egg size and its effects on offspring performance. Evolution 44: 279-294.

Smith C.C. \& Fretwell S.D. 1974: The optimal balance between size and number of offspring. Am. Nat. 108: 499-506.

SNyder W.E. \& Evans E.W. 2006: Ecological effects of invasive arthropod generalist predators. Annu. Rev. Ecol. Syst. 37: 95-122.

Sterns S.C. 1992: The Evolution of Life Histories. Oxford University Press, Oxford, $262 \mathrm{pp}$.

Sterns S.C. \& Hoekstra R.F. 2000: Evolution: An Introduction. Oxford University Press, New York, 381 pp.

Stewart L.A., Hemptinne J.-L. \& Dixon A.F.G. 1991: Reproductive tactics of ladybird beetles: relationships between egg size, ovariole number and developmental time. Funct. Ecol. 5: 380-385.

TAKAKURA K. 2004: Variation in egg size within and among generations of the bean weevil, Bruchidius dorsalis (Coleoptera, Bruchidae): effects of host plant quality and paternal nutritional investment. Ann. Entomol. Soc. Am. 97: 346-352.

TANigishi K. 1975: Comparative studies on sibling species of lady beetle, Harmonia axyridis and $\mathrm{H}$. yedoensis - differences in egg numbers per cluster. Paper presented at the $35^{\text {th }}$ Annual Meeting of the Entomol. Soc. Japan. [in Japanese].

THORNHILl R. \& Alcock J. 1983: The Evolution of Insect Mating Systems. Harvard University Press, Cambridge, 547 pp.

VANCE R.R. 1973: On reproductive strategies in marine benthic invertebrates. Am. Nat. 107: 339-352.

Wiebe K.L. \& Bortolotti G.R. 1994: Food supply and hatching spans of birds: energy constraints or facultative manipulation? Ecology 75: 813-823.

YASUdA H. \& OHNuma N. 1999: Effect of cannibalism and predation on the larval performance of two ladybird beetles. Entomol. Exp. Appl. 93: 63-67.

Yasuda H., Evans E.W., KajtTa Y., URakawa K. \& Takizawa T. 2004: Asymmetric larval interactions between introduced and indigenous ladybirds in North America. Oecologia 141: 722-731.

Received September 18, 2007; revised and accepted December 12, 2007 